\title{
Core Social Cognition
}

\section{Citation}

Spelke, Elizabeth S., Emily P. Bernier, and Amy E. Skerry. 2013. Core Social Cognition. In Navigating the Social World: What Infants, Children, and Other Species Can Teach Us, ed. Mahzarin R. Banaji and Susan A. Gelman, 11-16. New York: Oxford University Press.

\section{Published Version}

doi:10.1093/acprof:oso/9780199890712.003.0003

\section{Permanent link}

http://nrs.harvard.edu/urn-3:HUL.InstRepos:33980353

\section{Terms of Use}

This article was downloaded from Harvard University's DASH repository, and is made available under the terms and conditions applicable to Other Posted Material, as set forth at http:// nrs.harvard.edu/urn-3:HUL.InstRepos:dash.current.terms-of-use\#LAA

\section{Share Your Story}

The Harvard community has made this article openly available.

Please share how this access benefits you. Submit a story.

Accessibility 


\title{
1.3
}

\section{Core Social Cognition}

\author{
ELIZABETH S. SPELKE, EMILY P. BERNIER, AND AMY E. SKERRY
}

\begin{abstract}
$s$ a species, humans are distinguished by extraordinary capacities to learn and to socialize. A number of investigators of human cognitive development have proposed that these abilities are related: Our cognitive accomplishments are rooted in capacities and propensities for engaging with other people, learning from them, and navigating the social world (Csibra \& Gergely, 2011; Meltzoff, Kuhl, Movellan, \& Sejnowski, 2009; Tomasello, Carpenter, Call, Behne, \& Moll, 2005). These proposals align with recent research testifying to the surprising social acumen of human infants, much of which is described in this volume. Here, we ask about the cognitive architecture underlying these abilities: Are infants endowed with an integrated, fundamental system of social reasoning, and if so, is this system responsible for any of the unique accomplishments of our species? Although researchers have not yet answered these questions, we suggest that the field can begin to do so by probing the signature limits of infants' social capacities and mounting an interdisciplinary research effort to characterize those capacities.

This suggestion is inspired by the success of the last several decades of research on the development of perception and cognition. Research on human infants and young children has characterized infants' abilities to perceive and reason about objects (e.g., Baillargeon, 2004), actions (e.g., Woodward, 1998), number (e.g., Feigenson, Dehaene, \& Spelke, 2004), and geometry (e.g., Spelke, Lee, \& Izard, 2010). One of us has argued that this research provides evidence for five systems of core knowledge: knowledge of objects and their motions, of agents and their goal-directed actions, of number and the operations of arithmetic, of places in the navigable layout and their distances and directions from one another, and of geometrical forms and their length and angular relations. Herein, we unpack this core knowledge hypothesis by considering each of its three claims: that infants' knowledge is guided
\end{abstract}

by systems, that the systems are at the core of mature reasoning in these domains, and that these systems' computations give rise to knowledge. We then review how investigating boundary conditions and signature limits allowed the discovery and exploration of these systems across ages, species, and cultures. We suggest that understanding the nature of infants' social reasoning abilities will require a similar effort, and we discuss how such an approach could help to clarify current theories of human social cognitive development.

The core systems hypothesis states that infants represent and reason about the world in a way that accords with five specific, distinctive, and unitary bodies of principles. The principles are specific to a single domain (e.g., solid, bounded objects), because infants apply them to entities within the domain across wide variations in context and perceptual modality (for example, object principles are applied to tangible and hidden objects as well as to visible ones) and fail to apply them to perceptible entities outside the domain (Spelke, 1998). The five domains are distinct from one another, because the principles governing reasoning in each domain are different, producing systematic sets of double dissociations in infants' performance. Indeed, evidence for such dissociations has informed our understanding of mature cognition by suggesting that certain apparently unitary knowledge systems may in fact be founded on two distinct systems, as in the case of numerical and geometrical knowledge (Carey, 2009; Feigenson et al., 2004; Spelke et al., 2010). Finally, each set of principles forms a system, because when an entity is shown to behave in a manner that violates one principle, infants suspend the other principles (Huntley-Fenner, Carey, \& Solimando, 2002; Van de Walle, Rubenstein, \& Spelke, 1998).

The hypothesis that these are core systems is supported by research making systematic comparisons across species, across rearing conditions, and across human children and adults from 
different cultures or with differing access to the products of their culture. Diverse species represent objects, agents, number, places, and forms in the same ways human infants do, providing evidence for homologies in these cognitive capacities. In controlled-rearing experiments, animals have exhibited evidence of these representations on their very first encounters with entities in each of these domains (e.g., Chiandetti \& Vallortigara, 2011). These systems do not disappear over later human development but rather continue to exist in children (e.g., Cantlon, Safford, \& Brannon, 2011), appear in diverse cultures independently of formal education (e.g., Pica, Lemer, Izard, \& Dehaene, 2004), and are relatively preserved in children and adults with limited access to language and other symbol systems (for reviews, see Carey, 2009; Spelke, 2011).

Finally, the hypothesis that these are core systems of knowledge is supported by evidence that the systems support knowledge acquisition in children and the use of that knowledge in adults. Core notions of objects support intuitive reasoning about the physical world (Carey \& Spelke, 1994), core notions of agents allow us to infer goals from actions (Baker, Saxe, \& Tenenbaum, 2009), and core notions of number and geometry undergird both the learning and the performance of formal mathematics (Dehaene, 2009; Spelke, 2011). The behavioral signatures of each system can then support the tracing of these representational systems into the human brain, where the same cortical systems are engaged for nonsymbolic tasks tapping core knowledge systems and for symbolic tasks tapping abilities that are unique to educated humans (for examples in the domain of number, see Dehaene, 2009; Piazza, 2010).

Critically, these conclusions are supported not by the mere demonstration of infant cognitive abilities but by research probing the generality and limits of those abilities. The discovery of signature limits in infants' representations of objects, number, and geometry allowed for experiments that revealed the scope and boundaries of core knowledge in different domains and that tested whether the signature limits found in human infants were shared by nonhuman animals. The discovery of those common signatures, in turn, allowed for experiments on animals that tested systematically for effects of experience on the development of these systems (e.g., Chiandetti \& Vallortigara, 2011). The discovery of brain systems that show the same signatures allowed for experiments probing the neural mechanisms that support reasoning in these domains (e.g., Dehaene, 2009). And finally, the discovery of adult cognitive systems that show the same signatures allowed for experiments testing for the persistence of core systems over later development, their universality across cultures, and the roles played by those systems in mature reasoning (e.g., Scholl, 2002).

In this context, we can return to our first question: Do humans also possess a system of core social knowledge? Is infants' early engagement with the social world underpinned by a coherent and distinct set of principles that continues to guide their social reasoning throughout life? If there is a core system for social reasoning, we must identify the entities and events that constitute its domain and the inferences that it supports. What sort of information can and cannot be taken as input to this system, and what computations does it perform?

Here we consider evidence for three different answers to this question: Gergely and Csibra's hypothesis of an innate system for learning in pedagogical contexts (Csibra \& Gergely, 2011), Meltzoff's hypothesis of an innate system for matching one's own actions to the actions of others (Meltzoff, 2007), and Tomasello's hypothesis of an innate motivation to share attention and goals (Tomasello et al., 2005). We focus on these three theories for several reasons. First, each posits a system of social engagement that is unique to humans, and that promises to account for the development of our species' unique cognitive achievements. Second, each has generated an extensive body of research. Third, each is testable, we believe, through further research that follows the path of previous research on core cognition in other domains: research that defines the contours of the earliest developing social knowledge and then uses those contours in an interdisciplinary effort to probe the nature and development of this knowledge and its role in mature reasoning.

\section{NATURAL PEDAGOGY}

Following proposals by Premack and Premack (1996) and by Sperber and Wilson (1986), Gergely and Csibra suggest that our unique cultural heritage is a consequence of a species-specific adaptation for learning generalizable knowledge from communicative acts (Csibra \& Gergely, 2011). Under this theory, humans are predisposed to identify self-directed communicative acts from innately specified cues. These intentional communications are then interpreted via pedagogy-specific assumptions, which support inferences about 
generic properties of the objects and events that the communications highlight.

Vivid signatures of pedagogy, sometimes in the form of departures from optimal learning, come from studies of older children. For example, nonhuman apes learn useful ways to manipulate novel objects from skilled performers, ignoring irrelevant actions, but 3- and 4-year-old children tested in the same tasks copy manifestly irrelevant actions (Horner \& Whiten, 2005), as if trusting that the performer would not demonstrate actions unless they were causally relevant (Lyons, Young, \& Keil, 2007). Symmetrically, when an apparently knowledgeable adult deliberately demonstrates only a single action on a novel object, children are less likely to explore other properties of the object than they are when no demonstration occurred at all, when the demonstrator expresses ignorance of the object's functions, or when the pedagogical demonstration is interrupted. In conditions of full pedagogy, children evidently assume that a knowledgeable adult would have demonstrated other object functions if they existed (Bonawitz et al., 2011).

Recent work has begun to test for similar pedagogical assumptions in infancy, with tantalizing results. When a socially engaging adult looks at and speaks to an infant about some object or event, the infant learns different things about the world than when the same person simply acts on the object or event with no prior social engagement (Topal, Gergely, Miklosi, Erdohegyi, \& Csibra, 2008; Yoon, Johnson, \& Csibra, 2008). Nevertheless, it is unclear whether these effects reflect a pedagogical stance: Does social communication merely heighten or redirect infants' attention, or does it lead infants to make specific inferences about the evidence provided (Skerry, Lambert, Powell \& McAulliffe, in press)? This question could be addressed by testing for the effects of pedagogical learning found in older children. For example, will infants constrain their exploration of and learning about a new object based on an adult's completed pedagogical demonstration, and will this effect disappear if the communication is interrupted? Such experiments are necessary to distinguish pedagogy-specific inferences from lower level mechanisms for orienting attention to communicative adults. Only by linking learning in infants to learning in older children can we identify signatures by which to probe, through comparative research across ages and species, the nature, development, and evolution of this propensity to learn from communicative partners.

\section{NATURAL SIMILARITY}

Meltzoff (2007) has proposed that the crucial starting point for human social cognition is a propensity to learn from and affiliate with others who are represented as being like the self. Infants imitate the actions of others from birth (Meltzoff \& Moore, 1977), and by 14 months, prefer others who imitate them in turn (Meltzoff, 2007). These actions propel infants' learning both about and from their social partners, fostering the acquisition of language, cognitive skills, and social knowledge.

If a propensity for imitation is at the root of our species-specific social cognitive abilities, then aspects of this propensity should be unique to humans. Given that some imitative tendencies and preferences have been documented in nonhuman primates, both in neonates (Ferrari et al., 2006) and beyond (Horner \& Whiten, 2005), what are the specific features of human imitation that could support the rich social learning found in humans but not other species? One possibility, we suggest, is that a propensity to divide the social world into groups with common norms may serve to guide not only infants' own social interactions but also their understanding of the larger social landscape.

Human adults and older children not only behave like others with whom they wish to affiliate, and evaluate positively those who mirror their own behavior, but also conform to larger social groups and use evidence for conformity to reason about social relationships. Intriguing new work by Lindsey Powell suggests that by 8 months of age, human infants have a similar ability (Powell \& Spelke, 2011). Infants were shown animated displays depicting two social groups whose members engaged in two different characteristic actions, one per group. After observing the two actions performed by a subset of group members, infants viewed new group members performing the action of either their own or the other group. Infants looked longer at the inconsistent event in which a group member did not behave like others in its group. These findings suggest that conforming actions may have social meaning for 8-month-old infants, outside the context of their own social actions. However, further research is necessary to test this suggestion: What features of third-party interactions influence infants' expectations of behavioral similarity, and to what class of entities are these expectations applied? Once the origins and limits of these expectations are better defined, psychologists can turn to the broader questions of whether these early-developing capacities are 
seeds of our propensities for understanding and evaluating social conformity as adults, and whether they are unique to humans or shared by other animals.

\section{NATURAL COOPERATION}

Finally, Tomasello (2009) has proposed that the crucial differences between humans and other species stem from our unique biological predisposition to share psychological states with others. Evidence for this claim comes from a variety of studies examining the communicative and cooperative behaviors of toddlers. Within the second year of life children are predisposed to share attention and information (Liszkowski, Carpenter, Striano, \& Tomasello, 2006) and to engage in helpful actions toward others, even in the absence of any explicit request or reward (Warneken \& Tomasello, 2006). These observations raise the possibility that a core propensity to share mental states traces back to the beginning of human life.

Although active helping has not been reported systematically in the first year of life, a series of experiments on infants suggest that prosocial motivations may extend far back in development. Like Powell's studies, moreover, these experiments suggest that this propensity guides not only infants' own social interactions but also their understanding of the social interactions of others whom they observe as third parties. Building on research by Premack and Premack (1997), Hamlin and her collaborators presented infants with puppet shows in which two agents respectively help and hinder a third agent in achieving its goal. Six-month-old infants preferentially reached toward the agent who facilitated the goal over the agent who hindered it; 3-month-old infants showed a similar pattern of preferential looking at the helpful agent over the harmful one (Hamlin, Wynn, \& Bloom, 2010). These findings, and related findings with older infants, have been taken as evidence that infants appropriately valence the helper's and hinderer's behaviors, providing a foundation for social or moral evaluation (Hamlin, Wynn, \& Bloom, 2007).

These findings raise many questions concerning the conditions under which infants exhibit such preferences. One set of questions concerns the domain of these phenomena: Are they specific to completed and thwarted goal-directed actions, or might infants show preferences based on other social primitives such as harm or battery? A second set of questions concerns the inferences guiding infants' evaluations: Do infants infer the intentions, that is, the second-order goals, of helpers and hinderers? Do they impute emotional experiences to those who are in need of help, or to those who are wronged? As these questions are answered, investigators will gain tools for probing the role of these capacities in the development and practice of moral reasoning at later ages, and for reconstructing the evolution of these capacities through systematic comparisons across animal species.

These three examples illustrate a few of the more intriguing findings from the rich and growing body of research on social cognition in infancy. They begin to test general claims about the human mind while also providing ways to probe for the nature and development of human social knowledge. Nevertheless, none of these studies yet reveals whether a system of core social knowledge exists in infants. Moreover, this research does not reveal whether any of the social abilities and propensities found in young infants are unique to our species. Because all of the evidence for distinctively human patterns of pedagogy, conformity, and cooperation come from studies of older children, it is possible that these patterns develop from core social cognitive systems that humans share with other animals. Our uniquely human social propensities may result from the ways in which we build on these shared systems of core knowledge. To distinguish these possibilities, we must deepen our experiments and widen our view.

If a core system of social knowledge exists, it will be discovered by experiments that probe its contours, characterizing the input conditions that elicit specifically social reasoning and the principles that guide it (e.g., see Kinzler, Dupoux, \& Spelke, 2007). If it is unique to humans, then systematic differences should emerge when the same experiments are conducted on other species. If it is a guide to social reasoning throughout human life, then the same principles and limits should be found in humans in all cultures and at all ages. All of these findings will depend on the discovery of signature properties or profiles that can be used to identify common processes operating over development and across species, cultures, and levels of analysis.

If the system is an evolutionary adaptation to life in a social world, then evolutionary analyses focusing on the selection pressures shaping human evolution may provide insights into its limits and idiosyncrasies (e.g., Kurzban, Cosmides, \& Tooby, 2001). If the system lies at the center of our social reasoning as adults, then theories of mature human social reasoning, from 
social psychology, evolutionary and cultural anthropology (e.g., Henrich \& Broesch, 2011), and computational cognitive science (e.g., Shafto \& Goodman, 2008) may guide research into its foundations (e.g., Bonawitz et al., 2011). And if social cognitive development resembles development in other cognitive domains, then insights also will flow in the opposite direction: The search for the origins of social knowledge will shed light on the nature of mature social knowledge. As this volume attests, the study of social cognitive development is at an extremely exciting juncture, where all of the big questions are open, and where rich empirical and theoretical tools are available to address them.

\section{REFERENCES}

Baillargeon, R. (2004). Infants' physical world. Current Directions in Psychological Science, 13, 89-94.

Baker, C. L., Saxe, R., \& Tenenbaum, J. B. (2009). Action understanding as inverse planning. Cognition, 113, 329-349.

Bonawitz, E., Shafto, P., Gweon, H., Goodman, N. D., Spelke, E., \& Schulz, L. (2011). The double-edged sword of pedagogy: Instruction limits spontaneous exploration and discovery. Cognition, 120, 322-330.

Cantlon, J. F., Safford, K. E., \& Brannon, E. M. (2010). Spontaneous analog number representations in three-year-old children. Developmental Science, 13, 289-297.

Carey, S. (2009). The origin of concepts. New York: Oxford University Press.

Carey, S., \& Spelke, E. (1994). Domain-specific knowledge and conceptual change. In L. A. Hirshfeld \& S. A. Gelman (Eds.), Mapping the mind (pp. 169-200). Cambridge, UK: Cambridge University Press.

Chiandetti, C., \& Vallortigara, G. (2011). Intuitive physical reasoning about occluded objects by inexperienced chicks. Proceedings of the Royal Society B: Biological Sciences. doi: 10.1098/rspb.2010.2381 [ePub ahead of print].

Csibra, G., \& Gergely, G. (2011). Natural pedagogy as evolutionaryadaptation.PhilosophicalTransactions of the Royal Society B, 366, 1149-1157.

Dehaene, S. (2009). Origins of mathematical intuitions: The case of arithmetic. Annals of the New York Academy of Sciences, 1156, 232-259.

Feigenson, L., Dehaene, S., \& Spelke, E. S. (2004). Core systems of number. Trends in Cognitive Sciences, 8 , 307-314.

Ferrari, P.F., Visalberghi, E., Paukner, A., Fogassi, L., Ruggiero, A., \& Suomi, S. J. (2006). Neonatal imitation in rhesus macaques. PLoS Biology, 4(9), e302.
Hamlin, J., Wynn, K., \& Bloom, P. (2007). Social evaluation by preverbal infants. Nature, 450, 557-559.

Hamlin, J. K., Wynn, K., \& Bloom, P. (2010). 3-month-olds show a negativity bias in social evaluation. Developmental Science, 13, 923-939.

Henrich, J., \& Broesch, J. (2011). On the nature of cultural transmission networks: Evidence from Fijian villages for adaptive learning biases. Philosophical Transactions of the Royal Society, 366, 1139-1148.

Horner, V., \& Whiten, A. (2005). Causal knowledge and imitation/emulation switching in chimpanzees (Pan troglodytes) and children (Homo sapiens). Animal Cognition, 8, 164-181.

Huntley-Fenner, G., Carey, S., \& Solimando, A. (2002). Objects are individuals but stuff doesn't count: Perceived rigidity and cohesiveness influence infants' representations of small numbers of discrete entities. Cognition, 85, 203-221.

Kinzler, K. D., Dupoux, E., \& Spelke, E. S. (2007). The native language of social cognition. Proceedings of the National Academy of Sciences USA, 104, 12577-12580.

Kurzban, R., Tooby, J. \& Cosmides, L. (2001). Can race be erased? Coalitional computation and social categorization. Proceedings of the National Academy of Sciences USA, 98, 15387-15392.

Liszkowski, U., Carpenter, M., Striano, T., \& Tomasello, M. (2006). Twelve- and 18-month- olds point to provide information for others. Journal of Cognition and Development, 7, 173-187.

Lyons, D., Young, A., \& Keil, F. (2007). The hidden structure of overimitation. Proceedings of the National Academy of Sciences USA, 104, 19751-19756.

Meltzoff, A. N. (2007). "Like me": A foundation for social cognition. Developmental Science, 10, 126-134.

Meltzoff, A. N., Kuhl, P. K., Movellan, J., \& Sejnowski, T. J. (2009). Foundations for a new science of learning. Science, 325, 284-288.

Meltzoff, A. N., \& Moore, M. K. (1977). Imitation of facial and manual gestures by human neonates. Science, 198, 75-78.

Piazza, M. (2010). Neurocognitive start-up tools for symbolic number representation. Trends in Cognitive Science, 922, 1-10.

Pica, P., Lemer, C., Izard, W., \& Dehaene, S. (2004). Exactand approximate arithmeticin an Amazonian indigene group. Science, 306, 499-503.

Powell, L., \& Spelke, E. (2011, March 31-April 2). Eight-month-olds expect conformity from social but not nonsocial entities. Poster presented at the annual meeting of the Society for Research in Child Development, Montreal, Quebec. 
Premack, D., \& Premack, A. J. (1996). Why animals lack pedagogy and some cultures have more of it than others. In D. R. Olson (Ed.), The handbook of education and human development: New models of learning, teaching and schooling (pp. 302-323.). Malden, MA: Blackwell.

Premack, D., \& Premack, A. J. (1997). Infants attribute value to the goal directed actions of self-propelled objects. Journal of Cognitive Neuroscience, 9, 848-856.

Scholl, B. J. (Ed.). (2002). Objects and attention. Cambridge, MA: MIT Press.

Shafto, P., \& Goodman, N. (2008). Teaching games: Statistical sampling assumptions for pedagogical situations. In B. C. Love, K. McRae, \& V. M. Sloutsky (Eds.), Proceedings of the 30th Annual Conference of the Cognitive Science Society (pp. 1632-1636). Austin, TX: Cognitive Science Society.

Skerry, A. E., Lambert, E., Powell, L. J. and McAulliffe, K. (in press). The origins of pedagogy: developmental and evolutionary perspectives. Evolutionary Psychology.

Spelke, E. S. (1998). Where perceiving ends and thinking begins: The apprehension of objects in infancy. In A. Yonas (Ed.), Perceptual development in infancy. Minnesota Symposium on Child Psychology (Vol. 20, pp. 197-233). Hillsdale, NJ: Erlbaum.

Spelke, E. S. (2011). Natural number and natural geometry. In E. Brannon \& S. Dehaene (Eds.), Space, time, and number in the brain: Searching for the foundations of mathematical thought. Attention
\& Performance XXIV (pp. 287-313). Amsterdam: Elsevier..

Spelke, E. S., Lee, S. A., \& Izard, V. (2010). Beyond core knowledge: Natural geometry. Cognitive Science, 34(5), 863-884.

Sperber, D., \& Wilson, D. (1986). Relevance: Communication and cognition. Oxford, England: Blackwell.

Tomasello, M. (2009). Why we cooperate. Cambridge, MA: MIT Press.

Tomasello, M., Carpenter, M., Call, J., Behne, T., \& Moll, H. (2005). Understanding and sharing intentions: The ontogeny and phylogeny of cultural cognition. Behavioral and Brain Sciences, 28, 675-735.

Topal, J., Gergely, G., Miklosi, A., Erdohegyi, A., \& Csibra, G. (2008). Infants' perseverative search errors are induced by pragmatic misinterpretation. Science, 321(5897), 1831-1834.

Van de Walle, G., Rubenstein, J., \& Spelke, E. S. (1998). Infant sensitivity to shadow motions. Cognitive Development, 13, 387-419.

Warneken, F., \& Tomasello, M. (2006). Altruistic helping in human infants and young chimpanzees. Science, 311, 1301-1303.

Woodward, A. L. (1998). Infants selectively encode the goal object of an actor's reach. Cognition, 69, 1-34.

Yoon, J. M. D., Johnson, M. H., \& Csibra, G. (2008). Communication-induced memory biases in preverbal infants. Proceedings of the National Academy of Sciences USA, 105, 13690-13695. 